P53 (continued)

that motivate them while shopping, and food security status, using questions from the Perceived Nutrition Environment Measure Survey (NEMS-P) and the 6-item US Household Food Security Survey. A composite home food environment score was derived by summing availability and accessibility to healthy food and deducting unhealthy access and availability (range -15 to 19 , higher score means healthier home food environment). Analysis included multivariate linear regression models with the predictors and adjusting for demographic variables. Since this is an exploratory analysis, significance was set at $\mathrm{p}<0.10$.

Results: Being motivated by nutrition when shopping and food security were positively associated with the home food environment $(\beta=4.67, \mathrm{p}=0.028$ and $\beta=1.45$, $\mathrm{p}=0.058$ respectively). After controlling for socio-demographic characteristics, food security status was no longer significantly associated with improved home food environment but nutrition motivation remained significant $(\beta=4.89, \mathrm{p}=0.034)$.

Conclusion and Implications: Findings indicate that low-income individuals who consider nutrition important when grocery shopping have a significantly healthier home food environment. Nutrition education efforts to increase perceived importance of nutrition when grocery shopping may be a useful tool to improve the home food environment.

Funding: USDA

\section{P54 Community-based Policies and Support for Free Drinking Water in U.S. Municipalities}

Sohyun Park,PhD, spark3@cdc.gov, Centers for Disease Control and Prevention, 4770 Buford Highway, NE, Mail Stop F-77, Atlanta, GA 30341; Stephen Onufrak, PhD, Centers for Disease Control and Prevention; Cara Wilking, JD Legal Consultant; Angie Cradock, DSc, Harvard T.H. Chan School of Public Health

Objective: We examined community-level characteristics associated with free drinking water access policies in U.S. municipalities.

Study Design, Setting, Participants and Intervention: This cross-sectional study used data from a nationally representative survey of city managers/officials from 2,029 local governments conducted in 2014.

Outcome Measures and Analysis: Outcome measures were four policies regarding free drinking water access. Explanatory measures were population size, rural/urban status, census region, poverty prevalence, education, and racial/ethnic composition. We used multivariable logistic regression to test differences.

Results: For community planning, 56.3\% of local governments had at least one plan with a written objective to provide free drinking water in outdoor areas; municipalities in Northeast and South region and municipalities with $50 \%$ of non-Hispanic (NH) white were less likely to have a plan. For existing policies and funding, $59.2 \%$ had polices or budget provisions for free drinking water in parks and outdoor recreation areas. Municipalities in Northeast and South region were less likely to have such a policy or budget provision. For future community development, $9.3 \%$ provided incentives for placing drinking fountains in outdoor publicly accessible areas. For local building standards, $7.7 \%$ had a municipal plumbing code drinking fountain standard that differed from the statewide plumbing code; municipalities with lower proportion of $\mathrm{NH}$ whites were more likely to have it.

Conclusions and Implications: Free drinking water in parks and outdoor recreation areas are supported by written plans, a policy or budget provision in over half of municipalities. Drinking water promotion through development incentives or a more robust municipal plumbing code was rare. Encouraging the consumption of water instead of sugary drinks is a strategy to improve healthier diets and reduce disease risk.

Funding: None

\section{P55 Development of a Quantitative Measure of Community Readiness to Change for Health and Wellness}

Drew Hicks, BS, drew.hicks@sdstate.edu, South Dakota

State University, Box 2257A, SWG 441, Health and

Nutritional Sciences, Brookings, SD 57006;

Kendra Kattelmann, PhD, RDN, LN, FAND, South Dakota

State University

Objective: Recruiting community involvement in public health interventions is often met with varying degrees of success. Attitudes, resources, and leadership all impact a community's readiness to effect change. Assessing community readiness for change is vital to successful adoption of any health or wellness initiative. Past efforts in measuring community readiness have been largely limited. This study's objective is to develop an instrument capable of measuring the attitudes and ability of a college campus community towards adopting change in the realm of health and wellness.

Study Design, Setting, Participants: The Readiness for Community Wellness Survey was adapted from the Children's Healthy Living Program's Readiness to Collaborate Survey. The Delphi Technique will be used to recast measured domains to fit the needs of a college campus community health and wellness intervention. Cognitive interviews will be used to determine face validity. Survey measurements will then be evaluated against a modified Community Readiness Survey developed by the Minnesota Department of Human Services and Healthy Campus Environmental Assessments to determine external validity of the survey. Community and expert panel samples will be drawn from 8 U.S. college campuses involved with the USDA sponsored community-based participatory research project Fruved.

Results: Initial survey development has been completed. Six specific domains were identified as areas to assess for community readiness. Refinement and testing are currently in progress.

Continued on page $S 45$ 
P55 (continued)

Conclusions and Implications: Other attempts to measure community readiness for change have been explored, but no quantitative research methods for assessing a community's readiness for health and wellness change have been developed. A psychometrically sound and validated survey offers the possibility of accurate measuring community readiness for supporting successful institution of health and wellness interventions.

Funding: USDA

\section{P56 Development of the FoodCorps Healthy School Progress Report to Measure School Food Programming and Environment}

Pamela Koch, EdD, RD, pak14@tc.columbia.edu, Columbia University, 525 West 120th Street, Box137, New York, NY 10027; Randi Wolf, PhD, MPH, Columbia University; Matthew Graziose, PhD, Chobani; Raynika Trent, MS, MEd, Columbia University; Heewon Lee Gray, PhD, RD, University of South Florida; Isobel Contento, PhD, Columbia University

Objective: To develop a comprehensive tool to measure nutrition education, school garden experiences, healthy school meals, and schoolwide culture of health by revising a less comprehensive one.

Study Design, Setting, Participants: Collect process and outcome data and revise for 5 total versions. FoodCorps schools across United States (2014-15 and 2015-16 school years). FoodCorps service members, service site supervisors, school personnel, and stakeholders.

Outcome Measures and Analysis: Version 1: informed by literature review, interviews with 7 service members, and feedback from FoodCorps staff and board. Version 2: informed by 3 focus group phone calls and written comments from 35 stakeholders, including 6 school-based nutrition education researchers. Version 3: showed 11 high implementation schools had high HSPR scores and 9 "emerging" schools had low HSPR scores. Version 4: showed change scores for 298 FoodCorps schools completing the HSPR (Fall 2015 and Spring 2016). Conducted cognitive testing and process interviews with 9 FoodCorps Schools (3 people from each), analyzed for emerging themes. Version $5 \&$ Final HSPR: Incorporated results from prior versions.

Results: The HSPR is evidence-informed, has content and construct validity, and is sensitive to change. The tool measures FoodCorps programming in three areas of service: Hands-on Learning, Healthy School Meals, and Schoolwide Culture of Health. Average baseline scores were 42.7 (out of 100) and increasedeight points over one year. Schools that started with lower scores had bigger change scores.

Conclusions and Implications: The HSPR is a comprehensive measure of a healthy school environment and can be used by FoodCorps and other school-based nutrition education programs to assess levels of programming.

Funding: FoodCorps Inc.

\section{P57 Environmental Barriers to Healthful Dietary and Physical Activity Behaviors in the EFNEP Participant Population}

Lauren Rhoades, BS, MBA, lauren.rhoades@colostate.edu, Colorado State University, 502 West Lake Street, Fort Collins, CO 80526; Garry Auld, PhD, Colorado State University; Susan Baker, EdD

Objective: Direct education programs, including the Expanded Food and Nutrition Education Program (EFNEP), target personal barriers to enable behavior change. However, an opportunity exists to pursue a holistic policy, systems, and environment approach by targeting some environmental barriers (limited availability, high cost, safety, lack of transportation, and challenges caused by poor weather). The Western Regional Nutrition Education and Obesity Prevention Center of Excellence initiated a study to gather perspectives of EFNEP paraprofessional educators on how environmental barriers affect EFNEP participants and environmental changes which could be made to encourage more healthful participant behaviors.

Study Design, Setting, Participants: Ten telephone focus groups were held with fifty EFNEP educators from ten states during April and May of 2016.

Outcome Measures and Analysis: Focus groups were audio-recorded, transcribed, and independently coded by two reviewers to identify recurring themes.

Results: Educators provided valuable anecdotal evidence regarding environmental barriers and generated potential solutions for specific barriers based on examples from their communities. For example, potential solutions to the availability barrier include creating mobile farmers' markets, partnering with volunteer groups to provide free food delivery from food banks or retailers to areas with limited access, working with alternative retailers to offer produce in urban areas, providing seeds to encourage home gardening, or facilitating development of or participation in community gardens.

Conclusions and Implications: Results will guide additional research and inform development of environmental support activities for direct education programs reaching low-income participants. Activities will aim to empower participants to change environmental barriers in their micro-environments.

Funding: None

\section{P58 Good Choice Healthier Retail Initiative: Increasing Access and Appeal of Healthy Items in Rural Alabama}

Cecilia Tran, BS, Alabama Extension at Auburn University; Alicia Powers, PhD, arp0042@auburn.edu, Alabama Extension at Auburn University, 206 Duncan Hall, Auburn University, AL 36849; Barb Struempler, PhD, Alabama Extension at Auburn University; Sondra Parmer, PhD; Katie Funderburk, RD, MS 